\title{
Cold nanoindentation of germanium
}

L. Q. Huston, M. S. R. N. Kiran, L. A. Smillie, J. S. Williams, and J. E. Bradby

Citation: Appl. Phys. Lett. 111, 021901 (2017); doi: 10.1063/1.4993163

View online: https://doi.org/10.1063/1.4993163

View Table of Contents: http://aip.scitation.org/toc/apl/111/2

Published by the American Institute of Physics

\section{Articles you may be interested in}

Gallium kinetics on m-plane GaN

Applied Physics Letters 111, 022101 (2017); 10.1063/1.4993570

Rectangular photonic crystal nanobeam cavities in bulk diamond Applied Physics Letters 111, 021103 (2017); 10.1063/1.4992118

Announcement: Applied Physics Letters eliminates publication fees, effective 1 June 2017 Applied Physics Letters 111, 010201 (2017); 10.1063/1.4989855

Suppressed carrier density for the patterned high mobility two-dimensional electron gas at $\gamma-\mathrm{Al}_{2} \mathrm{O}_{3} / \mathrm{SrTiO}_{3}$ heterointerfaces

Applied Physics Letters 111, 021602 (2017); 10.1063/1.4993165

Enhancement of thermoelectric characteristics in AlGaN/GaN films deposited on inverted pyramidal Si surfaces Applied Physics Letters 111, 021902 (2017); 10.1063/1.4991969

Anomalous Seebeck coefficient observed in silicon nanowire micro thermoelectric generator Applied Physics Letters 111, 023105 (2017); 10.1063/1.4993150

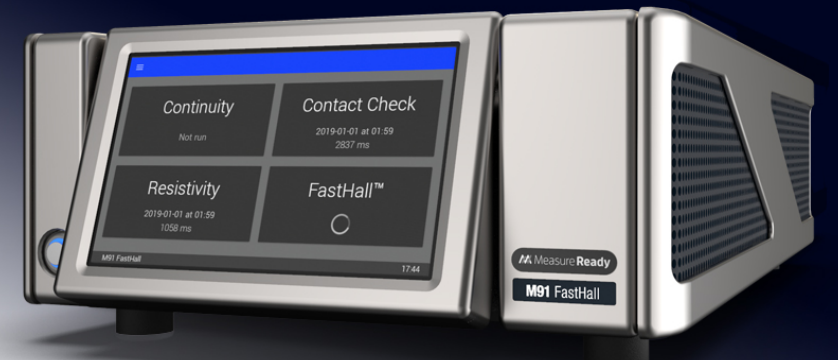

\section{Mak Measure Ready M91 FastHall ${ }^{\mathrm{TM}}$ Controller \\ A revolutionary new instrument for complete Hall analysis}




\title{
Cold nanoindentation of germanium
}

\author{
L. Q. Huston, ${ }^{\text {a) }}$ M. S. R. N. Kiran, ${ }^{\text {b) }}$ L. A. Smillie, J. S. Williams, and J. E. Bradby \\ Department of Electronic Materials Engineering, Research School of Physics and Engineering, The Australian \\ National University, Canberra, ACT 2601, Australia
}

(Received 4 May 2017; accepted 27 June 2017; published online 10 July 2017)

\begin{abstract}
Diamond cubic Ge is subjected to high pressures via nanoindentation at temperatures between $-45^{\circ} \mathrm{C}$ and $20^{\circ} \mathrm{C}$. The residual impressions are studied using ex-situ Raman microspectroscopy and cross-sectional transmission electron microscopy. The deformation mechanism at $20^{\circ} \mathrm{C}$ is predominately via the generation of crystalline defects. However, when the temperature is lowered, the analysis of residual indentation impressions provides evidence for deformation by phase transformation and formation of additional phases such as r8-Ge, hd-Ge, and amorphous Ge. Furthermore, these results show that at $0^{\circ} \mathrm{C}$ and below, dc-Ge will reliably phase transform via nanoindentation. Published by AIP Publishing. [http://dx.doi.org/10.1063/1.4993163]
\end{abstract}

The metastable phases of Ge formed using high pressure are of interest, as they have been predicted to have technologically significant properties. ${ }^{1,2}$ In particular, the rhombohedral (r8) and hexagonal diamond (hd) phases are predicted to be semiconductors with narrow band gaps, making them potentially useful for infrared detectors. ${ }^{1,2}$ Moreover, by extrapolating from predictions made for $\mathrm{Si}$, it is possible that the simple tetragonal (st12) structure of Ge may exhibit superconducting properties at around $30 \mathrm{~K}^{3}$

A standard method of forming such phases is compression using a high pressure diamond anvil cell (DAC). Ge is known to phase transform to the metallic beta-tin structure $(\beta-\mathrm{Sn}-\mathrm{Ge})$ at $\sim 10 \mathrm{GPa}^{4,5}$ Interestingly, $\beta$-Sn-Ge does not transform back to dc-Ge on decompression but rather to metastable phases such as st12-Ge, r8-Ge, and body centred cubic (bc8) Ge. ${ }^{6-9}$

Alternatively, similar high pressure phases in Ge have also been reported after nanoindentation. ${ }^{10-12}$ However, despite the fact that nanoindentation-induced phases broadly track those observed via DAC loading for $\mathrm{Si}$, this is not always the case for Ge. ${ }^{11,12}$ At room temperature, two different deformation mechanisms have been found to occur. ${ }^{13,14}$ One such deformation process is via the formation and propagation of "crystalline defects" such as slip and twinning. ${ }^{13,14}$ This process has been observed when indenting with both spherical and Berkovich tips. Alternatively, deformation via phase transformation appears to only occur during extreme loading conditions including fast loading rates, high loads, and very sharp tips. ${ }^{14-16}$ However, it has also been reported that even under such extreme conditions, phase transformations in $\mathrm{dc}-\mathrm{Ge}$ at room temperature are highly stochastic in nature and does not have a $100 \%$ probability of occurring. ${ }^{14}$ While the ease of phase transformation with a DAC can be attributed to the confinement of the material between the diamond anvils during loading, in the nanoindentation case, the material is loaded on a single axis and thus is not confined. Therefore, when slip and twinning occur, the local pressure is reduced under the indentation tip

\footnotetext{
a) larissa.huston@anu.edu.au

b) Present address: Department of Physics and Nanotechnology, SRM Research Institute, SRM University, Kattankulathur 603203, Chennai, Tamilnadu, India.
}

and the critical pressure for phase transformation may not be achieved. It is worth noting that, in contrast to dc-Ge, a-Ge can be reliably phase transformed presumably since the propagation of crystalline defects does not occur in the amorphous structure..$^{17-19}$

Although the nanoindentation of Si readily results in phase transformation at room temperature, ${ }^{11,12}$ altering the temperature has been shown to influence the deformation pathway. For example, above $\sim 150^{\circ} \mathrm{C}$, crystalline defect formation and propagation are the dominant deformation mechanisms of $\mathrm{Si}^{20}$ This is similar to the behavior of $\mathrm{Ge}$ at room temperature. Using $\mathrm{Si}$ as a model to understand similar behaviors in $\mathrm{Ge}$, we expect defect propagation to be suppressed by lowering the indentation temperature, thus favoring the phase transformation. As we will show in this letter, lowering the nanoindentation temperature of $\mathrm{Ge}$ to $0^{\circ} \mathrm{C}$ causes $100 \%$ of the indents to deform via phase transformation. Evidence of phase transformation is obtained from the analysis of the load-depth curves and characterisation of the residual impressions using both Raman microspectroscopy measurements and crosssectional transmission electron microscopy (XTEM).

Nanoindentation was performed using a Hysitron TI 900 TriboIndenter fitted with a custom-built cold stage. The stage was cooled by passing nitrogen gas (cooled by liquid nitrogen) through a copper block on which the sample was mounted. The temperature (measured on the sample with a Co-Al thermocouple) was controlled by varying the rate at which the nitrogen passed through the stage. The environment around the stage and transducer was purged with dry nitrogen gas in order to prevent the formation of ice on the surface of the sample. An array of 50 indents was made using a $1.3 \mu \mathrm{m}$ diameter spherical tip at the ambient temperature of $20^{\circ} \mathrm{C}$ and for a series of lower temperatures between $10{ }^{\circ} \mathrm{C}$ and $-45^{\circ} \mathrm{C}$. Both the loading and unloading times for each indent were $10 \mathrm{~s}$, and the maximum load was $10 \mathrm{mN}$. Each indentation impression was examined at ambient temperature using Raman microspectrometry with a $532 \mathrm{~nm}$ incident beam and a spot size of $\sim 1 \mu \mathrm{m}$. A laser intensity of $\sim 0.1 \mathrm{~mW}$ was chosen to avoid laser damage to the sample. Cross-sectional transmission electron microscopy (XTEM) was performed on selected indents using a Philips CM300 
transmission electron microscope. The XTEM samples were prepared using a FEI Helios Nanolab focused ion beam (FIB) system. Pt was first deposited to protect the surface of the area of interest. The sample was milled at $30 \mathrm{keV}$, and final thinning was done at $16 \mathrm{keV}$.

Figure 1 shows the nanoindentation behavior observed for dc-Ge at $20^{\circ} \mathrm{C}$ and $0{ }^{\circ} \mathrm{C}$. Using on a simple calculation based on the area of the tip, ${ }^{21}$ it would be expected that the contact pressure induced by the tip would first exceed $\sim 10 \mathrm{GPa}$ at less than $10 \%$ of the maximum load (assuming elastic deformation prior to phase transformation). In all cases, the loading portion of the indentation curve contains a number of very small discontinuities (labelled by arrows in Fig. 1) known as "pop-in" events, which are thought to be indicative of either slip/defect propagation and/or phase transformation to a dense metallic phase during loading. ${ }^{13}$ However, the unloading section of each of the curves shows different behaviors. A featureless unloading curve (left in Fig. 1) is observed for all indents at $20^{\circ} \mathrm{C}$, consistent with previous behavior where dc-Ge deforms via defect propagation at room temperature. ${ }^{13,22}$ At $0^{\circ} \mathrm{C}$, two different behaviors are observed. A pop-out event [as indicated on the middle curve of Fig. 1(a)] has previously been shown to be suggestive of a phase transformation from a dense metallic $(\beta-\mathrm{Sn})$ phase to less dense metastable crystalline phases on unloading. ${ }^{11,12,23}$ The elbow (right unloading curve) has been previously observed during indentation of $\mathrm{Si}$ in cases when the $\beta$-Sn metallic phase transforms to amorphous $\mathrm{Si}$ on fast unloading. ${ }^{11,23}$ Thus, we anticipate that an elbow is indicative of an a-Ge endphase. For an array of 50 indents, the number of each of these three unloading behaviors is presented in Fig. 1(b). At $0{ }^{\circ} \mathrm{C}, 32 \%$ of the indents exhibited a pop-out and $68 \%$ an elbow [Fig. 1(b)], and hence, it would appear that all 50 indentations result in phase transformation on loading (presumably to $\beta$-Sn-Ge) and a further phase transformation on unloading either to metastable crystalline Ge phases (pop-out) or a$\mathrm{Ge}$ (elbow). This is in clear contrast to the behavior at $20^{\circ} \mathrm{C}$ where there is no evidence of phase transformation.

The residual impressions of indents were further characterized by Raman and XTEM, with the results shown in

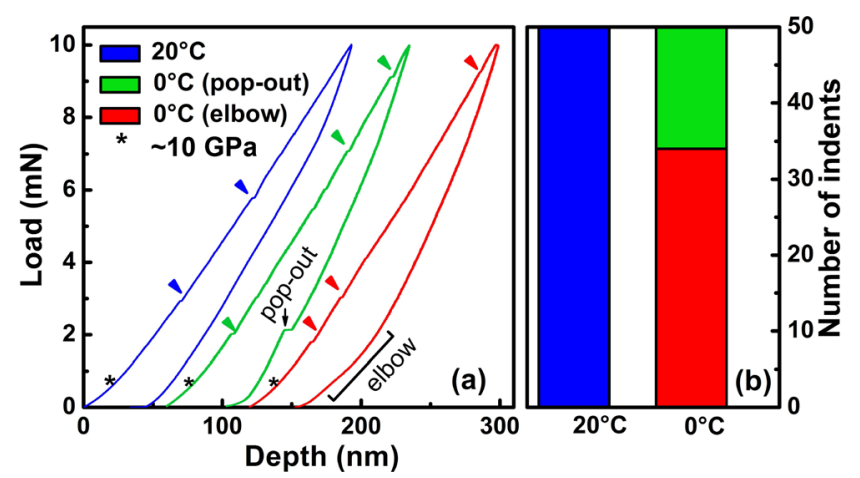

FIG. 1. A summary of different nanoindentation behaviors observed when indenting dc-Ge at $20^{\circ} \mathrm{C}$ and $0^{\circ} \mathrm{C}$. (a) A typical curve observed at $20^{\circ} \mathrm{C}$ and an example of curves displaying both "pop-out" and "elbow" events during unloading at $0^{\circ} \mathrm{C}$. Note for all three curves that a series of small pop-ins during loading (indicated with arrows) is observed, and the plots have been offset on the $\mathrm{X}$-axis for clarity. (b) The number of each of the behaviors observed at $20^{\circ} \mathrm{C}$ and $0{ }^{\circ} \mathrm{C}$, with blue indicating a featureless unloading curve, green indicating a "pop-out," and red indicating "elbow" behavior. The point where the contact pressure (assuming elastic behavior) reaches $10 \mathrm{GPa}$ is shown by an asterisk (*).
Fig. 2. At $20^{\circ} \mathrm{C}$, the Raman spectrum [as shown in Fig. 2(a)] indicates a dc-Ge peak (nominally centred at $301 \mathrm{~cm}^{-1}$ ) which is broadened towards higher wavenumbers compared with pristine dc-Ge (not shown). This is characteristic of disorder and stress in the dc-Ge within the indent volume and does not correspond to any other phases of Ge. ${ }^{18,19,24}$ The corresponding XTEM in Fig. 2(b) shows clear evidence for the formation of crystalline defects. The selected area diffraction pattern (SADP) analysis of the indented region [in the inset of Fig. 2(b)] shows predominately dc-Ge reflections.

The Raman and XTEM data corresponding to the indents that display a pop-out at $0{ }^{\circ} \mathrm{C}$ are shown in Figs. 2(c) and 2(d), respectively. The Raman spectrum shows both a dc-Ge peak at $301 \mathrm{~cm}^{-1}$ with a small shoulder to the left and some additional peaks. These additional peaks at 86, 94, 202, 225, and $249 \mathrm{~cm}^{-1}$ are known to be indicative of the presence of the $\mathrm{r} 8$ phase of Ge. ${ }^{19}$ These peaks were present in many but not all cases of pop-out behavior. It is proposed that r8-Ge formed in all of these indents, but in some cases, the $r 8$ phase had transformed into hd-Ge before the Raman characterisation was performed. Note that the Raman spectrum of hd-Ge contains a broad peak centered around $292 \mathrm{~cm}^{-1} .^{18}$ However, the strong dc-Ge peak at $301 \mathrm{~cm}^{-1}$ makes the identification of hd-Ge from the Raman spectrum difficult. The corresponding XTEM images of such pop-out indents [example in Fig. 2(d)] show evidence of a phase transformed zone within the indent impression, in addition to considerable disorder in the underlying dc-Ge with a similar morphology to the $20^{\circ} \mathrm{C}$ case. The transformed zone was found to predominately contain hd-Ge as concluded from the SADP [see circled reflections in the inset of Fig. 2(d) which correspond to hd-Ge]. This is consistent with previous observations where the r8-Ge phase was observed to transform to hd-Ge at ambient temperature within a few days. ${ }^{17,19}$

Finally, the Raman and XTEM data for indents at $0{ }^{\circ} \mathrm{C}$ which exhibit an elbow in the indentation unloading curves are shown in Figs. 2(e) and 2(f), respectively. In both the Raman spectrum and the XTEM image, there is evidence for a-Ge in the indent impression. This includes a well-defined transformation zone that contains a clear volume of a-Ge. Indeed, the SADP inset in Fig. 2(f) shows both dc-Ge reflections on the (110) zone axis and an a-Ge diffuse ring in the encircled region. It should be noted that the volume containing a-Ge [Fig. 2(f)] also contained some residual crystalline regions as can be seen by the small, darker regions in the uniform contrast amorphous zone, particularly directly under the surface. Note that trace amounts of hd-Ge were observed in the SADP of these samples, although the volumes of hd-Ge were insufficient for dark field imaging or to detect via Raman.

Thus, these results show that phase transformation in dc-Ge under uniaxial loading is favored by reducing the temperature. However, deformation via propagation of crystalline defects is not entirely suppressed as indicated by the underlying regions of crystalline defects shown in the $0{ }^{\circ} \mathrm{C}$ XTEM micrographs [Figs. 2(d) and 2(f)]. Following this observation, we propose the following mechanism for the deformation of dc-Ge. At the initial stage of loading into the plastic regime, extended defects may form at both $20^{\circ} \mathrm{C}$ and $0{ }^{\circ} \mathrm{C}$. At $20^{\circ} \mathrm{C}$, the formation and propagation of such defects occur at a rate that prevents the critical pressure for phase 

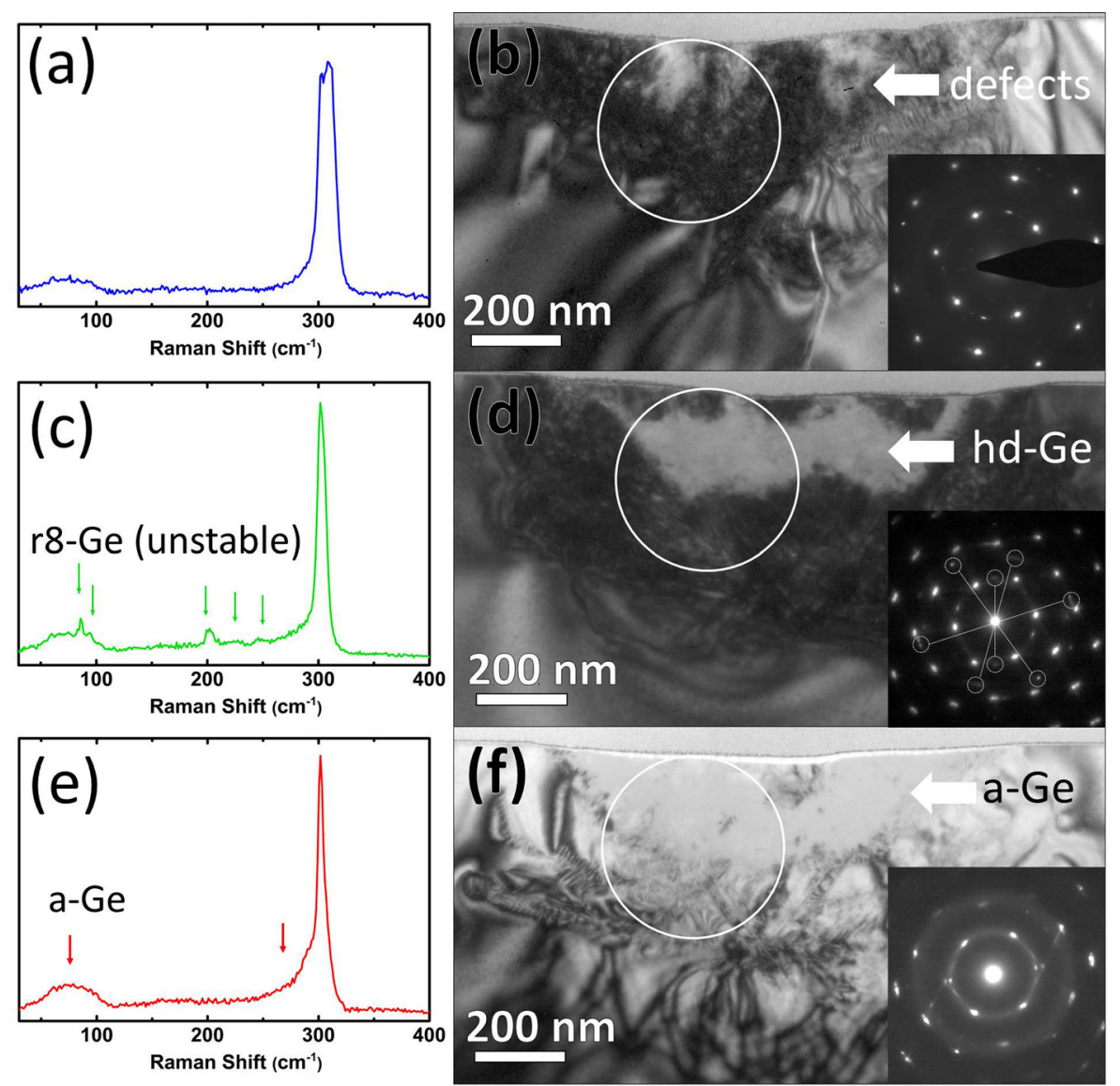

FIG. 2. Typical Raman spectra and XTEM images of indentation impressions formed at $20^{\circ} \mathrm{C}$ and $0^{\circ} \mathrm{C}$. (a) and (b) The indentation behavior at $20^{\circ} \mathrm{C}$. (c) and (d) Pop-out behavior observed at $0{ }^{\circ} \mathrm{C}$. (e) and (f) Elbow behavior observed at $0{ }^{\circ} \mathrm{C}$. Note that in the Raman spectra, phases other than diamond cubic are indicated with arrows. The regions circled in the XTEM images indicate the regions where the inset selected area diffraction patterns are collected from. In the pop-out case, reflections corresponding to the hd-Ge phase are circled. Note that the SADP rotation does not match image rotation and all SADPS are taken on the (1 110$)$ zone axis of the substrate. transformation from being reached. These defects remain on unloading. Hence, deformation at room temperature is via the propagation of crystalline defects and not phase transformation. This is shown schematically in Fig. 3(a). However, at $0{ }^{\circ} \mathrm{C}$, we suggest that deformation via slip/crystalline defect generation may still occur during loading but is slower than that at $20^{\circ} \mathrm{C}$. As a result, the local pressure directly under the indenter is able to increase to the critical phase transformation pressure despite some limited deformation via defect propagation. Upon unloading, this $\beta$-Sn-Ge transforms into either r8-Ge or a-Ge. This behavior is illustrated schematically in Fig. 3(b).

Given that the results show that even a modest decrease in temperature to $0^{\circ} \mathrm{C}$ changes the nanoindentation behavior from a situation where all indents exhibit deformation via the propagation of crystalline defects to one in which all indents undergo phase transformation, it is interesting to examine the behavior as temperature is further varied. Thus, 50 indents under the same indentation conditions were made at $10^{\circ} \mathrm{C}$, $-10^{\circ} \mathrm{C},-25^{\circ} \mathrm{C}$, and $-45^{\circ} \mathrm{C}$. The resultant deformation processes observed in both the unloading curve and Raman spectra are given in Fig. 4. At temperatures of $0{ }^{\circ} \mathrm{C}$ and below, complete phase transformation is observed to occur. However, the ratio of metastable crystalline to amorphous endphases decreases with temperature. The probability of $\mathrm{r} 8 / \mathrm{hd}-\mathrm{Ge}$ behavior is the highest at $0{ }^{\circ} \mathrm{C}$ and then decreases as temperature is lowered, while the probability of an amorphous endphase increases with decreasing temperature.

From these data, it is clear that the indentation temperature has a dramatic effect on the ability of dc-Ge to phase transform under point loading. To understand this, we turn to similar behavior previously observed in $\mathrm{Si}$. At room temperature, $\mathrm{Si}$ deforms under indentation predominately via phase transformation. ${ }^{11,12,25}$ When the temperature is increased to 150-200 ${ }^{\circ} \mathrm{C}$, slip/defect propagation then becomes the dominant deformation mechanism. ${ }^{20}$ This would suggest that the deformation of $\mathrm{Ge}$ at room temperature has similarities to

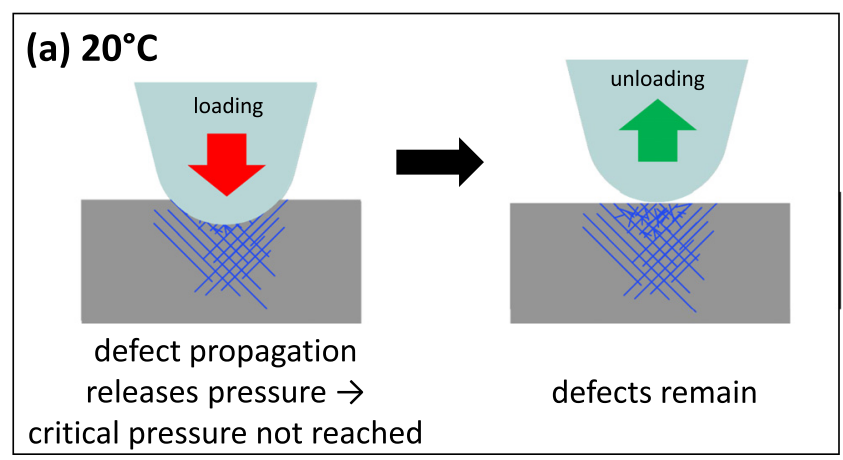

(b) $0^{\circ} \mathrm{C}$

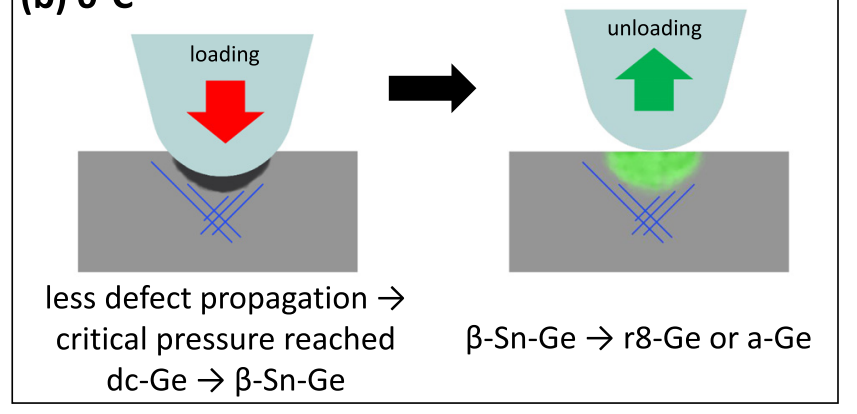

FIG. 3. A schematic showing the deformation behavior of dc-Ge via nanoindentation at (a) $20^{\circ} \mathrm{C}$ and (b) $0{ }^{\circ} \mathrm{C}$. 


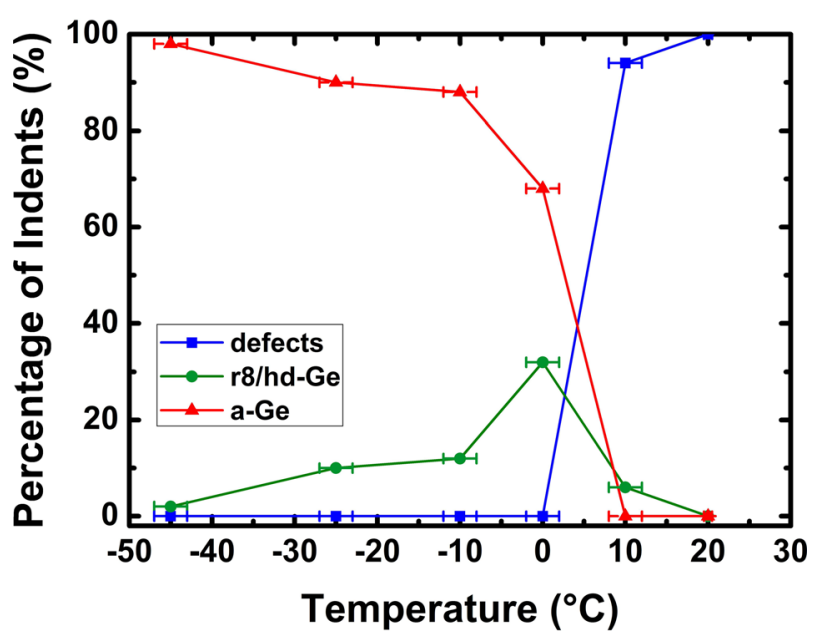

FIG. 4. The percentage of indents in dc-Ge out of 50 containing a particular endphase (defects, r8/hd-Ge or a-Ge) as a function of temperature.

those of Si at $150{ }^{\circ} \mathrm{C}$. Moreover, in $\mathrm{Si}$, it is well known that a high unloading rate $(\sim 1000 \mathrm{mN} / \mathrm{s})$ during nanoindentation at room temperature favors an amorphous endphase whilst a low unloading rate $(\sim 0.9 \mathrm{mN} / \mathrm{s})$ favors an $\mathrm{r} 8 / \mathrm{bc} 8-\mathrm{Si}$ endphase. ${ }^{26}$ This behavior has been attributed to the difficulty in nucleating $\mathrm{r} 8 / \mathrm{bc} 8-\mathrm{Si}$ from $\beta$-Sn-Si. ${ }^{27} \mathrm{In} \mathrm{Ge}$, we suggest that temperature is an important factor that inhibits the formation of r8-Ge. Clearly, in the temperature regime at and below $0{ }^{\circ} \mathrm{C}$, where slip and twinning are no longer the only deformation processes, thermal energy can assist with the nucleation of crystalline phases from $\beta$-Sn-Ge during unloading. However, when the temperature is below $-10^{\circ} \mathrm{C}$, the nucleation of the crystalline phases becomes energetically unfavorable, and the final structure is predominately amorphous. It should be noted that temperature may not be the only variable that influences the endphase following the nanoindentation of Ge. If low temperature Ge mirrors the behavior of room temperature $\mathrm{Si}$, then presumably, the unloading rate will also influence the endphase (or the percentage of $\mathrm{r} 8 \mathrm{-Ge}$ ) on unloading at low temperatures. However, we have not yet specifically investigate unloading rate effects.

Finally, it is worth commenting on the technological implications of these results. The data from this study offer an alternative method of making r8-Ge and hd-Ge. Hd-Ge is of technological interest due to its predicted direct band gap between $0.19 \mathrm{eV}$ and $0.55 \mathrm{eV}$, which makes it ideal for infrared devices. ${ }^{1,2}$ Indeed, if appropriate unloading conditions can be carried out to maximize the fraction of a crystalline hd-Ge endphase, as has been achieved for room temperature $\mathrm{Si}$, nanoindentation could be used to produce large arrays of this phase, facilitating the fabrication of devices that exploit its attractive properties. This approach will be especially viable if these results can be replicated with a larger tip sizes. It is also worth mentioning that similar phases of Si form via controlled scratching. ${ }^{28-30}$ The results of this study suggest that scratching of Ge might similarly be able to create larger regions of r8-Ge or hd-Ge under the right conditions (scratch rate and temperature), but increased shear stress under scratching could result in st12-Ge under some conditions. ${ }^{31,32}$
In conclusion, it is evident that lowering the temperature during nanoindentation favors phase transformation over defect-induced plastic deformation. This work provides the motivation for further studies to better understand the low temperature phase transformation kinetics in dc-Ge.

We acknowledge the support of the ANFF ACT Node and Centre for Advanced Microscopy at ANU in carrying out this research. This work was supported by the Australian Research Council. J.E.B. acknowledges the ARC for a Future Fellowship.

${ }^{1}$ B. D. Malone and M. L. Cohen, Phys. Rev. B 86, 054101 (2012).

${ }^{2}$ J. D. Joannopoulos and M. L. Cohen, Phys. Rev. B 7, 2644 (1973).

${ }^{3}$ B. D. Malone, J. D. Sau, and M. L. Cohen, Phys. Rev. B 78, 035210 (2008).

${ }^{4}$ S. Minomura and H. G. Drickamer, J. Phys. Chem. Solids 23, 451 (1962).

${ }^{5}$ J. C. Jamieson, Science 139, 762 (1963).

${ }^{6}$ V. V. Brazhkin, A. G. Lyapin, S. V. Popova, and R. N. Voloshin, Phys. Rev. B 51, 7549 (1995).

${ }^{7}$ R. J. Nelmes, M. I. McMahon, N. G. Wright, D. R. Allan, and J. S. Loveday, Phys. Rev. B 48, 9883 (1993).

${ }^{8}$ J. S. Kasper and S. M. Richards, Acta Cryst. 17, 752 (1964).

${ }^{9}$ F. P. Bundy and J. S. Kasper, Science 139, 340 (1963).

${ }^{10}$ G. M. Pharr, W. C. Oliver, and D. S. Harding, J. Mater. Res. 6, 1129 (1991).

${ }^{11}$ V. Domnich, Y. Gogotsi, and S. Dub, App. Phys. Lett. 76, 2214 (2000).

${ }^{12}$ J. E. Bradby, J. S. Williams, J. Wong-Leung, M. V. Swain, and P. Munroe, Appl. Phys. Lett. 77, 3749 (2000).

${ }^{13}$ J. E. Bradby, J. S. Williams, J. Wong-Leung, M. V. Swain, and P. Munroe, Appl. Phys. Lett. 80, 2651 (2002).

${ }^{14}$ D. J. Oliver, J. E. Bradby, J. S. Williams, M. V. Swain, and P. Munroe, J. Appl. Phys. 105, 126101 (2009).

${ }^{15}$ Y. Gogotsi, V. Domnich, S. N. Dub, A. Kailer, and K. Nickel, J. Mater. Res. 15, 871 (2000).

${ }^{16}$ J. Jang, M. J. Lance, S. Wen, and G. M. Pharr, Appl. Phys. Lett. 86, 131907 (2005).

${ }^{17}$ S. Deshmukh, B. Haberl, S. Ruffell, P. Munroe, J. S. Williams, and J. E. Bradby, J. Appl. Phys. 115, 153502 (2014).

${ }^{18}$ J. S. Williams, B. Haberl, S. Deshmukh, B. C. Johnson, B. D. Malone, M. L. Cohen, and J. E. Bradby, Phys. Status Solidi RRL 7, 355 (2013).

${ }^{19}$ B. C. Johnson, B. Haberl, S. Deshmukh, B. D. Malone, M. L. Cohen, J. C. McCallum, J. S. Williams, and J. E. Bradby, Phys. Rev. Lett. 110, 085502 (2013).

${ }^{20}$ M. S. R. N. Kiran, T. T. Tran, L. A. Smillie, B. Haberl, D. Subianto, J. S. Williams, and J. E. Bradby, J. Appl. Phys. 117, 205901 (2015).

${ }^{21}$ A. Fischer-Cripps, Nanoindentation (Springer-Verlag, New York, 2004).

${ }^{22}$ G. M. Pharr, W. C. Oliver, R. F. Cook, P. D. Kirchner, M. C. Kroll, T. R. Dinger, and D. R. Clarke, J. Mater. Res. 7, 961 (1992).

${ }^{23}$ V. Domnich and Y. Gogotsi, Rev. Adv. Mater. Sci. 3, 1 (2002).

${ }^{24}$ Z. Zhao, H. Zhang, D. Y. Kim, W. Hu, E. S. Bullock, and T. A. Strobel, Nat. Commun. 8, 13909 (2017).

${ }^{25}$ S. Wong, B. Haberl, J. S. Williams, and J. E. Bradby, J. Appl. Phys. 118, 245904 (2015).

${ }^{26}$ S. Ruffell, J. E. Bradby, and J. S. Williams, Appl. Phys. Lett. 89, 091919 (2006).

${ }^{27}$ S. Ruffell, J. E. Bradby, J. S. Williams, and P. Munroe, J. Appl. Phys. 102, 063521 (2007).

${ }^{28}$ S.-M. Jeong, H.-S. Oh, S.-E. Park, and H.-L. Lee, Jpn. J. Appl. Phys., Part 1 42, 2773 (2003).

${ }^{29}$ R. Gassilloud, C. Ballif, P. Gasser, G. Buerki, and J. Michler, Phys. Status Solidi A 202, 2858 (2005).

${ }^{30}$ S. Z. Chavoshi, S. C. Gallo, H. Dong, and X. Luo, Mater. Sci. Eng., A 684, 385 (2017).

${ }^{31}$ B. Haberl, M. Guthrie, B. D. Malone, J. S. Smith, S. V. Sinogeikin, M. L. Cohen, J. S. Williams, G. Shen, and J. E. Bradby, Phys. Rev. B 89, 144111 (2014).

${ }^{32}$ V. V. Brazhkin, A. G. Lyapin, S. V. Popova, and R. N. Voloshin, JETP Lett. 56, 152 (1992), http://www.jetpletters.ac.ru/ps/1283/article_19401.shtml. 